\title{
Premelting increases the rate of regelation by an order of magnitude
}

\author{
ALAN W. REMPEL, COLIN R. MEYER \\ Department of Earth Sciences, University of Oregon, Eugene, OR, USA \\ Correspondence: Alan W. Rempel <rempel@uoregon.edu>
}

\begin{abstract}
Glacier sliding over small obstacles relies on melting on their upstream sides and refreezing downstream. Previous treatments have appealed to 'pressure melting' as the cause of the spatial variations in melting temperature that drive this regelation process. However, we show that typical liquid pressure variations across small obstacles are negligible and therefore variations in ice pressure closely approximate variations in effective stress. For a given change in effective stress, the equilibrium melting temperature changes by an order of magnitude more than when the pressure of ice and liquid both change by an equal amount. In consequence, the temperature gradients that drive heat flow across small obstacles are larger than previously recognized and the rate of regelation is faster. Under typical conditions, the transition wavelength at which ice deformation and regelation contribute equally is of $m$-scale, several times longer than previous predictions, which have been reported to underestimate field inferences.
\end{abstract}

Keywords: ice dynamics, ice physics, glacier modeling, melt-basal, subglacial processes

\section{INTRODUCTION AND SCALING ARGUMENTS}

Textbook treatments of glacier sliding based on pioneering work by Weertman (1957); Nye (1969); Kamb (1970) describe how glacier motion is facilitated through a combination of ice deformation over larger bed obstacles, and the process of melting and refreezing across smaller obstacles that is referred to as regelation (Cuffey and Paterson, 2010). In these treatments, the rate of regelation is limited by the conduction of latent heat across the obstacle in response to changes in melting temperature that accompany variations in ice pressure. Here, we follow the classical scaling arguments for the rate of regelation (e.g. Fowler, 2011) to demonstrate that the permeability between the ice and the obstacle, which limits the rate at which water can flow, requires that significant local differences arise between the ice and liquid pressures. When the pressure in a liquid differs from that imposed on its adjacent solid (more precisely, the first invariant of the stress tensor), the theory of premelting describes how equilibrium is achieved at a temperature that differs from the bulk melting temperature that holds when the pressures in the two phases are the same (Dash and others, 2006). Taking premelting into account, we modify the Kamb (1970) treatment of sliding and demonstrate both that the rate of regelation is faster than previously thought, and that the effects of pressure melting and refreezing are typically of negligible importance in comparison to melting and refreezing sourced to variations in effective stress.

We begin with the scaling for conduction of the latent heat due to the refreezing of water. The rate of regelation $v_{R}$ is linked to the time required to transport volumetric latent heat $\rho_{i} \mathcal{L}$ by conduction at effective thermal conductivity $K$ from the downstream side of small obstacles, where the melting temperature is elevated slightly, to the upstream side, where higher ice pressures cause the melting temperature to be depressed. For obstacles of wavelength $\lambda$, scaling considerations suggest that energy transport requires

$$
v_{R} \sim-\frac{2 K \Delta T}{\rho_{i} \mathcal{L} \lambda},
$$

where $\Delta T$ is the (negative) difference in melting temperature between the upstream and downstream sides. Conventionally, this melting temperature difference is linked to the (positive) difference in ice pressure $\Delta P_{i}$ between the upstream and downstream sides through the Clapeyron slope, i.e. $\Delta T=$ $-C_{0} \Delta P_{i}$, with

$$
C_{0}=\frac{T_{\mathrm{m}}}{\rho_{i} \mathcal{L}} \frac{\rho_{l}-\rho_{i}}{\rho_{l}},
$$

where $T_{\mathrm{m}} \approx 273 \mathrm{~K}$ is a reference melting temperature, and $\rho_{i}$ and $\rho_{l}$ are the ice and liquid densities; $C_{0}$ is sensitive to the air-content dissolved in the liquid (e.g. Röthlisberger and Lang, 1987; Clarke, 2005), with reported values ranging between $9.8 \times 10^{-8} \mathrm{~K} \mathrm{~Pa}^{-1}$ (saturated) and $7.4 \times 10^{-8} \mathrm{~K}$ $\mathrm{Pa}^{-1}$ (pure). Where this description of phase equilibrium is valid, it follows that the regelation velocity scales as

$$
v_{R} \sim \frac{2 K C_{0} \Delta P_{i}}{\rho_{i} \mathcal{L} \lambda}
$$

As noted above, the equilibrium between liquid water and ice tracks along the Clapeyron slope of the bulk phase diagram only when the ice and liquid pressures are equal to each other along their common interface so that $\Delta P_{i}=\Delta P_{l}$. Considerations of mass conservation constrain the difference in liquid pressure between the upstream and downstream sides that is required to drive transport of liquid with viscosity $\mu$, whereupon

$$
\Delta P_{I} \sim \frac{\rho_{i} \mu v_{R} \lambda}{\rho_{l} \Pi} .
$$

Here, the resistance to flow at low Reynolds number is characterized by effective permeability $\Pi$; for example, Darcy flow 
through a porous medium with the permeability $\Pi$ or an equivalent Poiseuille flow through a liquid film of thickness $\delta$, which can be described with $\Pi=\delta^{2} / 12$. Substituting in for the regelation velocity scaling from the energy transport argument that led to Eqn (1) suggests that

$$
\Delta P_{I} \sim-\frac{2 \mu K \Delta T}{\rho_{l} \mathcal{L} \Pi}
$$

Since the melting temperature difference between the upstream and downstream sides falls along the Clapeyron slope so that $\Delta T=-C_{0} \Delta P_{i}$ only when $\Delta P_{l}=\Delta P_{i}$, the special case in which regelation is driven by pressure melting alone holds when the permeability to liquid transport is

$$
\Pi \sim \Pi_{0}=\frac{2 \mu K C_{0}}{\rho_{l} \mathcal{L}} .
$$

Taking nominal values of $\mu=1.8 \times 10^{-3}$ Pa s, $K=2 \mathrm{~W} \mathrm{~m}^{-1} \mathrm{~K}^{-1}$, $\mathcal{L}=3.3 \times 10^{5} \mathrm{~J} \mathrm{~kg}^{-1}$, and treating the case of pure water so that $\rho_{l}=10^{3} \mathrm{~kg} \mathrm{~m}^{-3}$ and $C_{0}=7.4 \times 10^{-8} \mathrm{~K} \mathrm{~Pa}^{-1}$, implies that $\Pi_{0} \approx 1.6 \times 10^{-18} \mathrm{~m}^{2}$, which in the case of thin film flow corresponds with $\delta_{0} \approx 4.4 \mathrm{~nm}$.

Permeabilities as low as $\Pi_{0}$ are uncommon in surficial geological materials. Moreover, when the proximity between ice and mineral substrates $\delta$ is reduced below the micron scale, the intermolecular forces that cause premelting (e.g. Dash and others, 1995, 2006; Wettlaufer and Worster, 2006) enable significant load transfers between the ice and mineral surfaces so that the ice pressure exceeds the liquid pressure, with their difference identified as the effective stress $N=P_{i}-P_{l}$. Hence, for the idealized case of sliding over a completely impermeable bed with all liquid transport confined within an interfacial film, we infer that the film thickness $\delta_{0}$ implied by mass-balance constraints is inconsistent with the central assumption of regelation by pressure melting alone, which holds that $P_{i}=P_{I}$ and $N=0$. Instead, the generalized Clapeyron equation (see Fig. 1) must be invoked to describe the changes in melting temperature that result from variations in both liquid pressure and effective stress as (cf. Rempel, 2008, Eqn (1))

$$
\Delta T \approx-C_{0}\left(\Delta P_{l}+\frac{\rho_{l}}{\rho_{l}-\rho_{i}} \Delta N\right),
$$

where each of these quantities (i.e. $\Delta T, \Delta P_{l}, \Delta \mathrm{N}=\Delta P_{i}-\Delta P_{l}$ ) represents the difference between values on the upstream and downstream sides of bed obstacles. Substituting Eqn (7) into Eqn (1) gives a regelation velocity of

$$
v_{R} \sim \frac{\rho_{l}}{\rho_{l}-\rho_{i}} \frac{2 K C_{0} \Delta P_{i}}{\rho_{i} \mathcal{L} \lambda}\left[1-\frac{\rho_{i} \Delta P_{l}}{\rho_{l} \Delta P_{i}}\right] .
$$

In the typical case where $\Pi \gg \Pi_{0}$ from Eq. (6) efficient liquid drainage is expected to diminish liquid pressure variations so that $\Delta P_{i} \sim\left(\Pi / \Pi_{0}\right) \Delta P_{l} \gg \Delta P_{l}$, and in this limit Eqn (8) implies a regelation velocity of

$$
v_{R} \sim \frac{\rho_{l}}{\rho_{l}-\rho_{i}} \frac{2 K C_{0} \Delta P_{i}}{\rho_{i} \mathcal{L} \lambda}
$$

For pure water, Eqn (9) suggests that $v_{R}$ is $\rho_{/} /\left(\rho_{l}-\rho_{i}\right) \approx 12$ times faster than predicted by conventional treatments, which rely upon temperature differences that result from

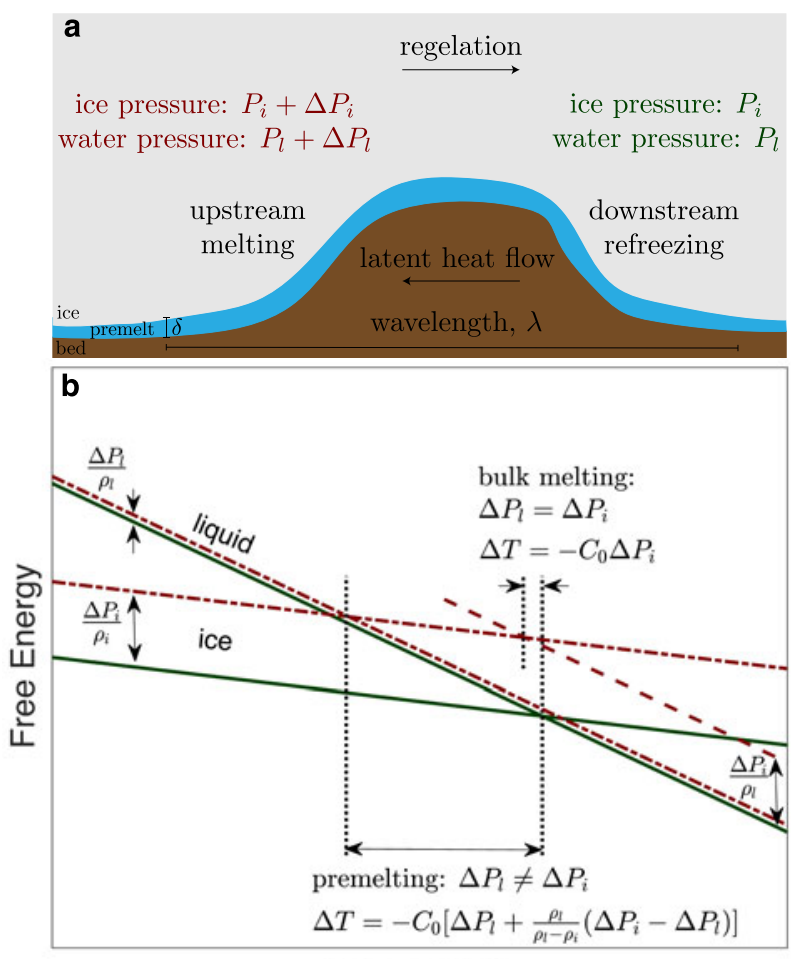

Temperature

Fig. 1. Generalized Clapeyron conditions for regelation. (a) Schematic of regelation past a bump. (b) Gibbs free energy per molecule of ice and liquid water. The ice lines are more shallow because ice has a lower specific entropy than liquid. Separate lines for a given substance correspond to different pressures. Intersections of ice and liquid lines correspond to phase equilibria. Consider the intersection of the two green lines (e.g. at the pressure conditions on the downstream side of a bump): at temperatures below the phase equilibrium temperature, ice has the lower free energy, and above the melting temperature, liquid prevails due to its lower free energy. The red lines show the free energy in each phase when pressure is elevated as labeled. If the ice and liquid pressures both increase by the same amount, the melting temperature decreases along the Clapeyron slope with the intersection between the dashed and the dot-dashed red lines. If, however, the ice and liquid pressures are perturbed by different amounts (e.g. at the pressure conditions on the upstream side of a bump), the melting temperature decrease can be much larger, as shown by the intersection of the two dot-dashed red lines.

pressure melting alone (cf. Eqn (3)). The enhanced regelation speed predicted by Eqn (9) is the primary finding of this work.

\section{LINEARIZED ICE FLOW OVER WAVY TOPOGRAPHY}

Further insight can be gained by revisiting the detailed analysis provided by Kamb (1970) for regelation (at velocity $v_{R}$ ) and 'plastic' deformation (at velocity $v_{\mathrm{p}}$ ) over a rough glacier bed, while making the minor extensions required to describe how the water pressure $P_{\text {I }}$ varies independently from the ice pressure $P_{i}$. The details of this calculation are provided in the Supplementary Information. The interface we consider is described by

$$
z_{0}(x, y)=\frac{A}{4 \pi^{2}} \iint_{-\infty}^{\infty} a(h, k) \mathrm{e}^{i(h x+k y)} \mathrm{d} h \mathrm{~d} k
$$

for area element $A$, Fourier spectrum a, and horizontal wavenumbers $h$ (along flow) and $k$ (perpendicular to flow). We 
account for the transport of heat and ice of viscosity $\eta$ as in Kamb (1970), while assuming that liquid flow follows Darcy's law and that the interface is at its melting temperature everywhere, as given by Eqn (7). This results in a modified version of Kamb's expression relating the shear stress $\tau$ and sliding velocity $v=v_{R}+v_{\mathrm{P}}$, given by

$$
\tau=\frac{A}{4 \pi^{2}} 2 \eta v \iint_{-\infty}^{\infty} h^{2} \ell \mathrm{a}^{2}(h, k) \frac{\ell_{0}^{2}+\ell_{\Pi}^{2}}{\ell^{2}+\ell_{0}^{2}+\ell_{\Pi}^{2}} \mathrm{~d} h \mathrm{~d} k .
$$

where $\ell^{2}=h^{2}+k^{2}$, and the characteristic wavenumbers $\ell_{0}$ and $\ell_{\Pi}$ are defined by

$$
\ell_{0}^{2}=\frac{\rho_{l}-\rho_{i}}{\rho_{l}} \frac{\rho_{i} \mathcal{L}}{4 \eta C_{0} K} \text { and } \ell_{\Pi}^{2}=\frac{\rho_{i}^{2} \mu}{\rho_{l}^{2} 2 \eta \Pi} .
$$

The length scale $2 \pi / \ell_{0}$ is the transition wavelength at which the conduction of latent heat required by regelation and the deformation of ice over an obstacle offer equal resistance, whereas $2 \pi / \ell_{\Pi}$ marks distances for which the liquid transport needed to accommodate regelation imposes a significant impediment. Since reasonable permeability estimates satisfy $\Pi \gg \rho_{i} \Pi_{0} /\left[\left(\rho_{l}-\rho_{i}\right)\right]$, a comparison of the limiting wave numbers under such conditions yields $\ell_{\Pi}^{2} \ll \ell_{0}^{2}$, implying that liquid transport plays a negligible role in restricting regelation. Hence, as in conventional treatments of regelation due to pressure melting (Weertman, 1957; Nye, 1969; Kamb, 1970; Gudmundsson, 1997), the relative importance of plastic deformation to regelation in controlling sliding speed depends on a comparison between the topographic wavelength $2 \pi / \ell$ and the transition wavelength $2 \pi / \ell_{0}$. Importantly, the transition wavelength is governed by considerations of heat flow, and because liquid transport is relatively efficient, variations in ice pressure produce commensurate variations in effective stress. The resultant perturbations to the melting temperature (see Fig. 1) cause the transition wavelength to be $\sqrt{\left(\rho_{l}-\rho_{i}\right) / \rho_{l}} \approx 3.5$ times larger than would be expected if the phase behavior were governed by pressure melting alone. For example, adopting the nominal parameter values given above and assigning an ice viscosity of $\eta=10^{-13}$ Pa s leads to $2 \pi / \ell_{0} \approx 3 \mathrm{~m}$.

Of particular interest here, for interfaces with roughness only at wavelengths small enough that $\ell^{2} \gg \ell_{0}^{2}+\ell_{\Pi}^{2}$ so that plastic deformation is negligible (i.e. $v \approx v_{R}$ ), Eqn (11) reduces to

$$
\tau \approx \frac{A}{4 \pi^{2}} \frac{\rho_{i} \mathcal{L} V}{2 C_{0} K}\left(\frac{\rho_{l}-\rho_{i}}{\rho_{l}}+\frac{\rho_{i}}{\rho_{l}} \frac{\Pi_{0}}{\Pi}\right) \iint_{-\infty}^{\infty} \frac{h^{2} \mathrm{a}^{2}(h, k)}{\ell} \mathrm{d} h \mathrm{~d} k,
$$

where $\Pi_{0}$ is defined as in Eqn (6). In the special case where $\Pi=\Pi_{0}$ we note that the term in parentheses on the right tends to unity so that the relationship between $\tau$ and $v$ predicted by treatments that set $P_{i}=P_{l}$ and consider pressure melting alone (e.g. Weertman, 1957; Nye, 1969; Kamb, 1970) is recovered; with $\Pi \gg \Pi_{0}$, for a given basal shear stress $\tau$ the regelation velocity predicted by Eqn (13) is a factor of $\rho_{/} /\left(\rho_{l}-\rho_{i}\right) \approx 12$ greater.

\section{IMPLICATIONS}

In attempting to validate aspects of his treatment of glacier sliding Kamb (1970, see §17) compared his predicted transition wavelength to field observations and found evidence suggesting that conventional theory underestimated $2 \pi / \ell_{0}$. In revising expectations of the transition wavelength upwards by a factor of 3.5, the current treatment may yield an explanation for this discrepancy and is promising for efforts to map basal roughness at the resolution of the Weertman (1957) 'controlling obstacles'. Perhaps more important than such quantitative improvements is the enhanced recognition of the importance of the effective stress in basal dynamics, and particularly basal thermodynamics. Though the role of subglacial water pressure in controlling sliding behavior through its effects on subglacial mechanics is well established in a variety of contexts (Lliboutry, 1968; Iken and Bindschadler, 1986; Tulaczyk and others, 2000; Schoof, 2010), its importance in affecting the ice-liquid equilibrium temperature and premelting (Dash and others, 2006) has arguably been under-appreciated (Alley and others, 1997).

Here, we have demonstrated that liquid transport near the basal interface (primarily as a consequence of premelting rather than the density difference that determines the sign of the Clapeyron slope) causes an order of magnitude increase to the speed of regelation. Indeed, because effective stress variations drive melting and refreezing in the same qualitative manner in any system where a solid is wetted by its own melt (i.e. premelting takes place), this suggests a more general role for regelation as a viable mechanism for accommodating slip over small obstacles in diverse geological systems where pressure melting cannot occur because the melt is less dense than its solid. In the glacial context, we anticipate that effective-stress-driven changes in melting temperature are likely to be common causes of basal melting and refreezing, possibly even supercooling (e.g. Röthlisberger and Lang, 1987; Alley and others, 1998; Lawson and others, 1998; Creyts and Clarke, 2010). Evidence has been given that the thermo-mechanical feedbacks made possible by premelting and variations in effective stress are instrumental in limiting both the range of observed glacier driving stresses (Meyer and others, 2018) and the occurrence of regions amenable to stick-slip behavior (Lipovsky and others, 2019). This adds further emphasis to the importance of basal liquid transport for understanding glacier dynamics (e.g. Clarke, 2005; Iverson, 2010).

\section{ACKNOWLEDGEMENTS}

We thank Tim Creyts, Neal Iverson, Brad Lipovsky, Elisa Mantelli, Brent Minchew, Kiya Riverman, Olga Sergienko and Christian Schoof for discussions; we appreciate the guidance of scientific editor lan Hewitt, and the constructive comments of Martin Lüthi and an anonymous reviewer; we also acknowledge financial support from NSF-1603907.

\section{SUPPLEMENTARY MATERIAL}

The supplementary material for this article can be found at https://doi.org/10.1017/jog.2019.33

\section{REFERENCES}

Alley RB and 5 others (1997) How glaciers entrain and transport basal sediment: physical constraints. Quat. Sci. Rev., 16(9), 1017-1038 
Alley RB, Lawson DE, Evenson EB, Strasser JC and Larson GJ (1998) Glaciohydraulic supercooling: a freeze-on mechanism to create stratified, debris-rich basal ice: II. Theory. J. Glaciol., 44(148), $563-569$

Clarke GKC (2005) Subglacial processes. Annu. Rev. Earth Planet. Sci., 33, 247-276

Creyts TT and Clarke GKC (2010) Hydraulics of subglacial supercooling: theory and simulations for clear water flows. J. Geophys. Res., 115, F03021 (doi: 10.1029/2009JF001417)

Cuffey KM and Paterson WSB (2010) The physics of glaciers, 4th edn. Elsevier, San Francisco

Dash JG, Fu H and Wettlaufer JS (1995) The premelting of ice and its environmental consequences. Rep. Prog. Phys., 58(1), 115

Dash JG, Rempel AW and Wettlaufer JS (2006) The physics of premelted ice and its geophysical consequences. Rev. Mod. Phys., 78(3), 695

Fowler A (2011) Mathematical geoscience, vol. 36. Springer Science \& Business Media, London

Gudmundsson GH (1997) Basal-flow characteristics of a non-linear flow sliding frictionless over strongly undulating bedrock. J. Glaciol., 43(143), 80-89

Iken A and Bindschadler RA (1986) Combined measurements of subglacial water pressure and surface velocity of Findelengletscher, Switzerland: conclusions about drainage system and sliding mechanism. J. Glaciol., 32(110), 101-119

Iverson NR (2010) Shear resistance and continuity of subglacial till: hydrology rules. J. Glaciol., 56(200), 1104-1114
Kamb B (1970) Sliding motion of glaciers: theory and observation. Rev. Geophys., 8(4), 673-728

Lawson DE and 5 others (1998) Glaciohydraulic supercooling: a freeze-on mechanism to create stratified, debris-rich basal ice: I. Field evidence. J. Glaciol., 44(148), 547-562

Lipovsky BP and 5 others (2019) Glacier sliding, seismicity, and sediment entrainment. Ann. Glaciol. (doi: 10.1017/aog.2019.24)

Lliboutry L (1968) General theory of subglacial cavitation and sliding of temperate glaciers. J. Glaciol., 7, 21-58

Meyer CR, Downey AS and Rempel AW (2018) Freeze-on limits bed strength beneath sliding glaciers. Nat. Commun., 9(1), 3242

Nye JF (1969) A calculation on the sliding of ice over a wavy surface using a Newtonian viscous approximation. Proc. R. Soc. Lond. A, 311(1506), 445-467

Rempel AW (2008) A theory for ice-till interactions and sediment entrainment beneath glaciers. J. Geophys. Res., 113(F1), f01013

Röthlisberger $\mathrm{H}$ and Lang $\mathrm{H}$ (1987) Glacial hydrology. In Gurnell AM and Clark MJ, eds. Glacio-fluvial sediment transfer: an alpine perspective. John Wiley and Sons, New York, 207-284

Schoof C (2010) Ice sheet acceleration driven by melt supply variability. Nature, 468(7325), 803-806

Tulaczyk S, Kamb WB and Engelhardt HF (2000) Basal mechanics of Ice Stream B, West Antarctica: 1. Till mechanics. J. Geophys. Res., 105(B1), 463-481

Weertman J (1957) On the sliding of glaciers. J. Glaciol, 3(21), 33-38

Wettlaufer JS and Worster MG (2006) Premelting dynamics. Ann. Rev. Fluid Mech., 38(1), 427-452

MS received 2 January 2019 and accepted in revised form 29 April 2019 